\title{
Towards Hardware Optimal Neural Network Selection with Multi-objective Genetic Search
}

\author{
O. Krestinskaya, \\ Nazarbayev University \\ Nur-Sultan, Kazakhstan
}

\author{
K. Salama, \\ King Abdullah University of Science and \\ Technology (KAUST), Thuwal, Saudi Arabia
}

\author{
A. P. James \\ Maker Village; IIITM; Kerala Govt.; \\ Kerala, India;Email: apj@ieee.org
}

\begin{abstract}
The selection of hyperparameters and circuit components for optimum hardware implementation of a neural network is a challenging task, which has not been automated yet. This work proposes the method for the selection of optimum neural network architecture and hyperparameters using genetic algorithm based on the hardware-related performance metrics, such an on-chip area, power consumption, processing time and robustness to hardware non-idealities, and focus on memristorbased analog network architecture. The experimental results show that the proposed approach allows to select the optimum architecture based on the designers' preferences.
\end{abstract}

Index Terms-Memristor, Neural Networks, Analog circuit, Hyperparameter selection, Genetic algorithm

\section{INTRODUCTION}

The conventional neural network hyperparameter selection approaches do not consider the hardware related performance metrics when searching for the optimum network architecture [1]-[4] and the selection of the optimum circuit level architecture for hardware implementation of the neural networks is performed manually. In this paper, we propose to automate the selection of neural network architecture using evolutionary genetic algorithm [5]-[9]. A multiobjective optimization approach is followed for the selection of the optimum neuro-memristive hardware implementation taking into account hardware-based performance metrics, such as an on-chip area, maximum power consumption, and processing time. The objective function takes into account memristor nonidealities [10]-[14] including limited number of stable resistive states [15]-[17], resistance variation [18]-[21], possibility of device failure [11], [22], [23] and memristor aging [24], [25].

\section{Methodology AND RESUlts}

1) Methodology: In this work, we focus on the hyperparameter optimization for analog memristive neural network implementations. We consider the selection of four hyperparameters of fully connected network: (1) number of hidden layers, (2) number of neurons in hidden layers, (3) activation function of the hidden layers and (4) activation function of the output layer. To reduce the complexity, we keep the number of neurons and activation functions in all hidden layers the same.

The analog circuit components of the considered architecture are illustrated in Fig. 1. We focus on the network implementation using 1T1R memristive crossbar (Fig. 1 (a)) with difference amplifier readouts to represent positive and

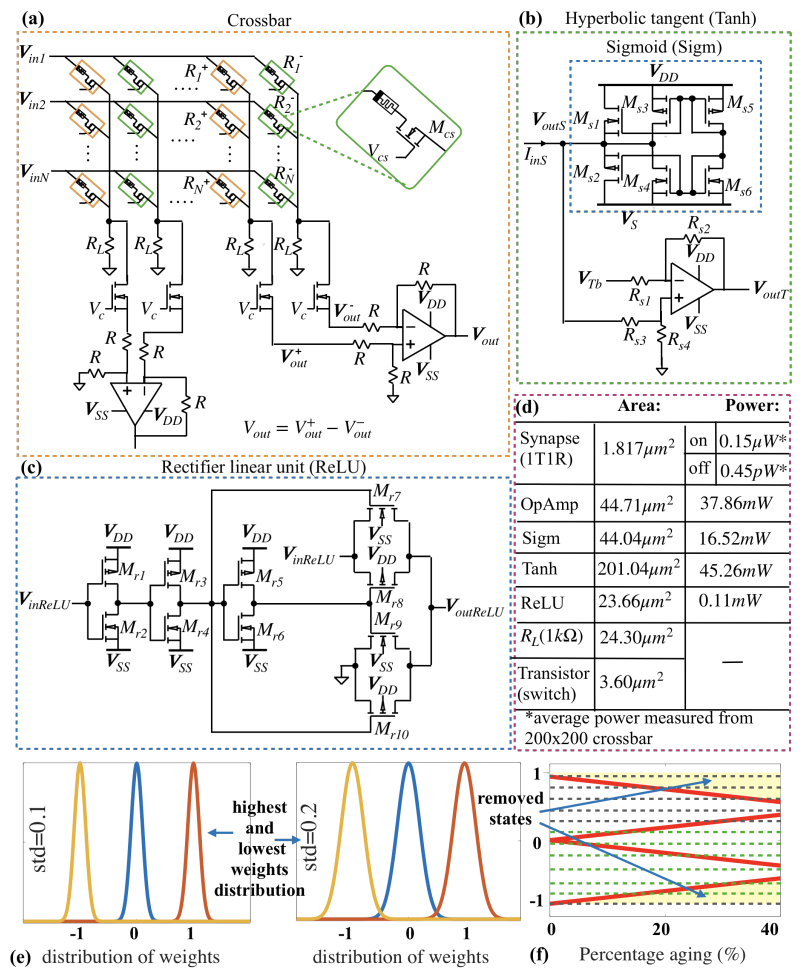

Fig. 1: (a) 1T1R memristive crossbar array; (b-c) Tanh, Sigmoid and ReLU activation function circuits; (d) on-chip area and power consumption of separate components; (e) distribution of weights considering conductance variation; (f) aging effect in memristive devices.

negative memristive weights. The crossbar columns are read sequentially and switched using transistors in the columns. Fig. 1 (b-c) illustrates hyperbolic tangent (Tanh), rectifier linear unit $(R e L U)$ and sigmoid (Sigm) activation functions [26] considered in the hyperparameter selection. Fig. 1 (d) shows on-chip area and maximum power consumption of the selected activation functions and crossbar components verified by SPICE simulations.

The hyperparameters selection and the ways to include hardware parameters to the selection of the architecture are shown in Alg. 1, and related parameters are summarized in the footnote ${ }^{1}$. The main idea of the genetic algorithm is to create a random population (Alg.1 (2-4)), a set of random 
networks, from the hyperparameter vector, and evolve and mutate (Alg.1 (19-22)) the population after each generation maximizing the objective function (Alg.1 (18)) until reaching the optimum solution. We set an objective function $S$ as a weighted summation of the scores of accuracy, area, power and processing time, normalized between 0 and 1 , where " 1 " refers to minimum area/power/time and maximum accuracy (Alg.1 (17)). The normalization considers the minimum and maximum area and power of the architecture based on the list of selected hyperparameters. The experiments can include more parameters in objective function calculation when considering the effect of different hardware non-idealities. The on-chip area is calculated according to Eq. 1, taking into account the separate difference amplifier and activation function required for the crossbar columns. Eq. 2 shows the calculation of maximum power consumption, which refers to the maximum possible power consumption during the READ operation of a crossbar column considering that CMOS components of the OFF columns are completely disconnected from power supply. The processing time is calculated as $t=T\left(n l+n_{\text {out }}\right)$ considering sequential processing of the crossbar columns. The explanation of the parameters of Eq. 1-2 is listed as footnote ${ }^{1}$.

$$
\begin{aligned}
& A=n\left(n_{\text {in }}+n_{\text {out }}+(l-1) n\right) A_{\text {syn } 2}+ \\
& +\left(n_{\text {out }}+\ln \right) A_{\text {perif }}+n l A_{a H}+n_{\text {out }} A_{a O} \\
& P=\max \left(n_{\text {in }} P_{\text {on } 2}+n_{\text {in }}(n-1) P_{o f f 2}+P_{o p}+P_{a H}\right. \text {, } \\
& \left.n P_{\text {on } 2}+\left(n_{\text {out }}-1\right) n P_{\text {off } 2}+P_{o p}+P_{a O}\right)
\end{aligned}
$$

\section{otherwise:}

In the experiments, we consider the robustness of the architecture to memristor non-idealities, such as limited number of stable resistive states (quantization) [15], [17], resistance variation [18], [27], possibility of memristor failure [22] and memristor aging with time [24], [25]. The limited number of resistive states is considered quantizing the weights to $L$ states between $W_{\min }$ and $W_{\max }$ (Alg.1 (8)). The resistance variation is performed using Gaussian distribution function $G_{d}()$ with mean $\mu=0$ and varying standard deviation $s t d=\sigma$ (Alg.1 (10)). The example for the distribution of a quantized minimum and maximum memristive weight is shown in Fig. 1 (e). The memristor failure is performed by setting $f \%$ of random memristors in a crossbar to $R_{o n}$ or $R_{\text {off }}$ states (Alg.1 (12)). The effect of memristor aging is evaluated by removing $a \%$ of highest and lowest resistive states in the device shown in Fig. 1 (f).

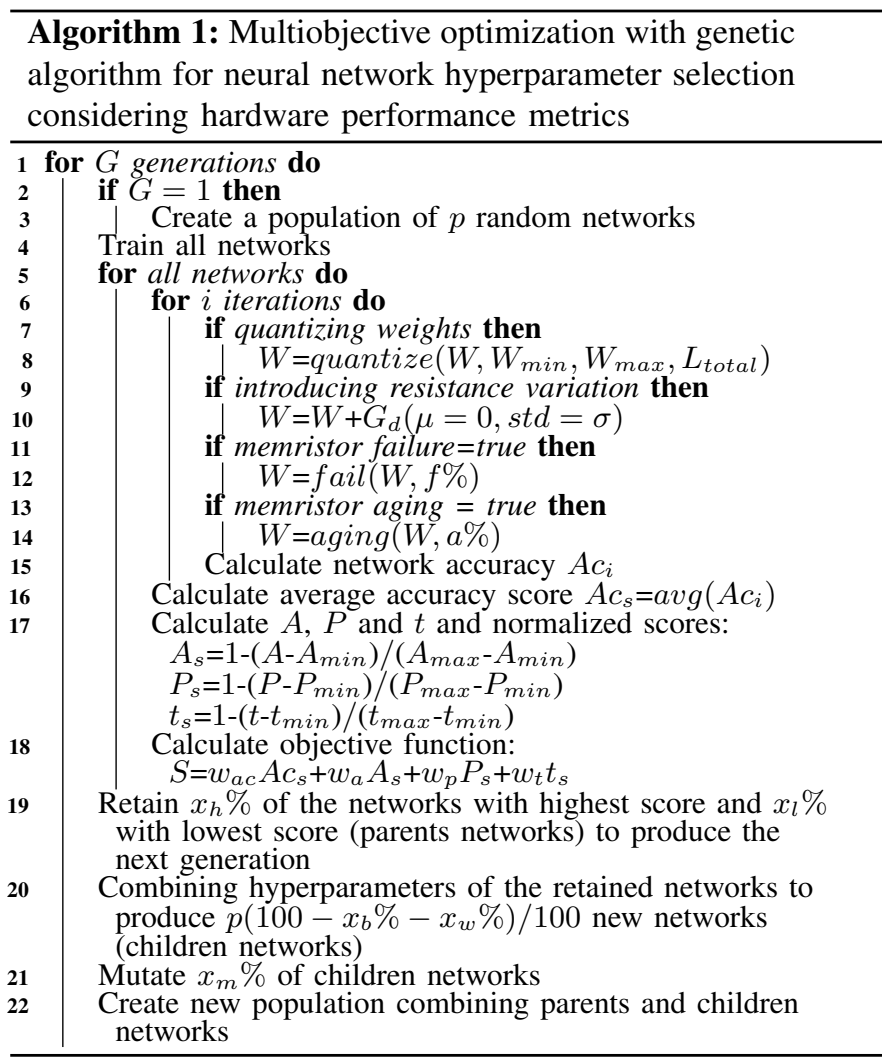

2) Simulation results: The experiments are performed for MNIST [28] and Fashion-MNIST [29] databases. The hyperparameter optimization in the neural network architecture was performed for $n=[64,128,256,512,768,1024], l=$ $[1,2,3,4]$ and $[$ Sigm, Tanh, ReLU] activation functions for hidden and output layers. During the training, a categorical cross-entropy loss function and Adamax optimizer [30] have been used for 25 epoch for MNIST and 70 epoch for FashionMNIST databases. The algorithm parameters were set to $G=10, p=10, x_{b}=40 \%, x_{w}=10 \%, x_{m}=20 \%$. The hardware parameters are illustrated in Fig. 1 (d) based on 180nm CMOS circuits and $\mathrm{WO}_{x}$ memristive devices [31] simulated with Knowm MSS (Multi-Stable Switch) memristor model [32] with $T=80 \mu s$ [33] and $A_{m e m}=0.0169 \mu m^{2}$ [34].

The simulation results for optimization of different parameters and comparison of the optimized solutions for MNIST and Fashion-MNIST databases are illustrated in Fig. 2. Fig.

\footnotetext{
${ }^{1} G, p$ - number of generations, number of networks in a population; $W$ - memristive weights $\left(W_{\min / \max }\right.$ - minimum/maximum weight); $i$ - number of iterations for non-ideal networks accuracy calculation; $A c_{s}$ - average network accuracy $A c_{s}=a v g\left(A c_{i}\right)$ calculated as quad an average of accuracy scores $A c_{i}$ of each iteration $i$; $L$ total - number of stable weight levels; if $w_{\text {min }}<0, L_{\text {total }}=2 L$, where $L$ - number of stable conductance states in memristor; $f$, $a$ - percentage of memristor failure, percentage of memristor aging; $n, l$ - number of neurons, number of layers in the network; $n_{i n}, n_{\text {out }}$ - number of input and output neurons in the network; $A, P, t$ - network area, maximum power and processing time; $A_{s}, P_{s}, t_{s}$ - scores of area, power and time normalized between 0 and $1 ; S$ - total score of the objective function; $w_{a c / a / p / t}-$ weights of accuracy/area/power/time in total score calculation; $P_{a H / a O}$ - area and power of activation functions of hidden/output layer; $P_{t a} / r e / s i-$ area and power of Tanh/ ReLU/ Sigmoid; $P_{\text {on } 2 / o f f 2}$ - power per two $1 \mathrm{~T} 1 \mathrm{R}$ synapses (single weight) in "on"/"off" state: $P_{\text {on } 2 / o f f 2}=2 P_{o n / o f f} ; A_{s y n 2}-$ area of two $1 \mathrm{~T} 1 \mathrm{R}$ synapses $A_{s y n 2}=2\left(A_{m e m}+A_{t}\right)$, where $A_{\text {mem }}$ - memristor area, $A_{t}$ - transistor area; $A_{o p}, P_{o p}$ - area and power of opamp-based difference amplifier; $A_{r l}, A_{s w}$ - area of load resistor / column switch; $A_{p e r i f}$ - area of peripheral circuits $A_{\text {perif }}=2 A_{r l}+2 A_{s w}+A_{o p} ; T$ - time to "read" the output of a single crossbar column; $x_{h / l} \%$ - percentage of retained networks with the highest/lowest score; $x_{m} \%$ - percentage of mutated networks when creating next population; $P_{\max / \min }, A_{\max / \min }, t_{\max / \min }-$ maximum/minimum power/area/time of a particular hyperparameter optimization setup, calculated applying min/max number of layers and neurons $n=n_{\min / \max }$ and $l=l_{\min } / \max$ to Eq. (1-2).
} 

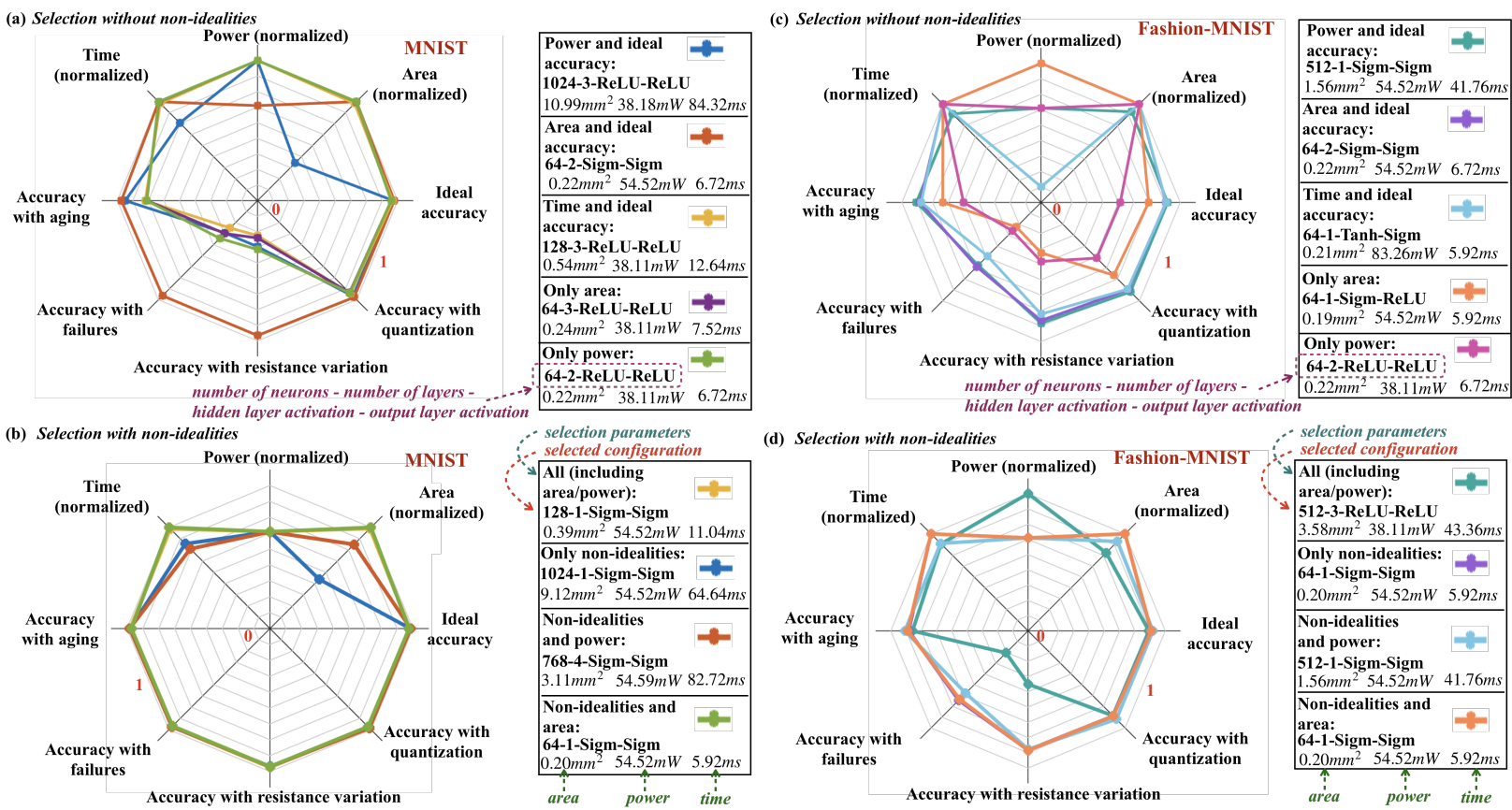

Fig. 2: Selection of the architecture for MNIST (a-b) and Fashion-MNIST (c-d) databases based on performance accuracy and hardware parameters $(\mathrm{a}, \mathrm{c})$ with ideal memristive devices, and $(\mathrm{b}, \mathrm{d})$ considering memristor non-idealities.

$2(\mathrm{a}, \mathrm{c})$ illustrates the performance of selected networks based on ideal memristive devices for 5 different variations of the objective function. For example, if the selection parameters are "power and ideal accuracy", the objective function of genetic algorithm is set to $S=0.5 P_{s}+0.5 A c_{s}$. The corresponding simulation results (Fig. 2 (a), blue line) show that the selected architecture has 1024 neurons in all hidden layers, 3 layers and $R e L U$ activation functions in both hidden and output layers, and is optimal in maximum power consumption per READ cycle $(P=38.18 \mathrm{~mW})$ and ideal accuracy $(\approx 95 \%)$, while the parameters that are not considered, such as the on-chip area $\left(A=10.99 \mathrm{~mm}^{2}\right)$, processing time $(t=84.32 \mathrm{~ms})$ and accuracy scores considering memristor non-idealities $(\approx 30 \%)$ are not optimized. Overall, Fig. 2 (a,c) show that the consideration of memristor non-idealities is important in the optimization, and the optimized ideal accuracy/area/power does not imply the optimal performance of the architecture with memristor non-idealities.

The selected architectures and the performance metrics of the experiments considering memristor non-idealities, such as $L=16, \sigma=0.1, f=2 \%, a=10 \%$ and $i=20$, are shown in Fig. 2 (b,d). Overall, the selected architectures show more optimal results for non-ideal cases, while on-chip area and power are larger than in the ideal simulations, and the algorithm tends to select the architectures with Sigm activation and larger $n$ (more neurons). The simulation results considering "all" parameters imply the optimization for 7 parameters (power/area/time + performance accuracy scores for 4 types of non-idealities) considering $S=\frac{1}{7} P_{s}+\frac{1}{7} A_{s}+$ $\frac{1}{7} t_{s}+\frac{1}{7} \sum_{j=1}^{4} A c_{s j}$. We considered the simplest example, where all selection parameters have equal weights, while these weights can be distributed unevenly based on the output preferences.

The proposed approach can be applied for the selection of the optimal architecture considering particular non-ideality, e.g. selection of the architecture based on the limited number of stable resistive states, and can illustrate how memristor nonidealities affect the selection of an optimal architecture. The simulation results for Fashion-MNIST database, considering quantization for $L=[2,4,16,64]$, resistance variation $\sigma=[0.05$, $0.1,0.15,0.2]$ and memristor aging $a=[5,10,20,25] \%$, are illustrated in Fig. 3 (a-c). The normalized octagon area (between 0 and 1) illustrates how optimal is the architecture, considering all performance metrics, where the architecture with higher total score has larger octagon area. The results for the quantization (Fig. 3 (a)) show that when the number of stable resistive states is reduced (2,4 states), the selected architectures (Fig. 3 (a), light blue and orange lines) have larger octagon area $(\approx 0.85)$ and has smaller number of neurons $(n=256)$ and layers $(l=1)$. This shows that it is impossible to improve the accuracy, when the number of states is small $(L=2$ or $L=4)$; and the accuracy with quantization is $80 \%$ for $L=2$ and $86 \%$ for $L=4$. Therefore, the algorithm does not tend to select the large $n$ and $l$, as when $L=64$ or $L=16$, where accuracy still can be increased to $\approx 90 \%$ by increasing $n$ and $l$. While, the experiment with resistance variation (Fig. 3 (b)) is opposite, and selected $l$ and $n$ increase with the increase of $\sigma$. This allows to achieve the accuracy of $\approx 85-86 \%$ for $\sigma=0.05-0.1$ (Fig. 3 (b), cian and purple lines) and $\approx 70-80 \%$ for $\sigma=0.15-0.2$ (Fig. 3 (b), light blue and orange lines). The algorithm considering 

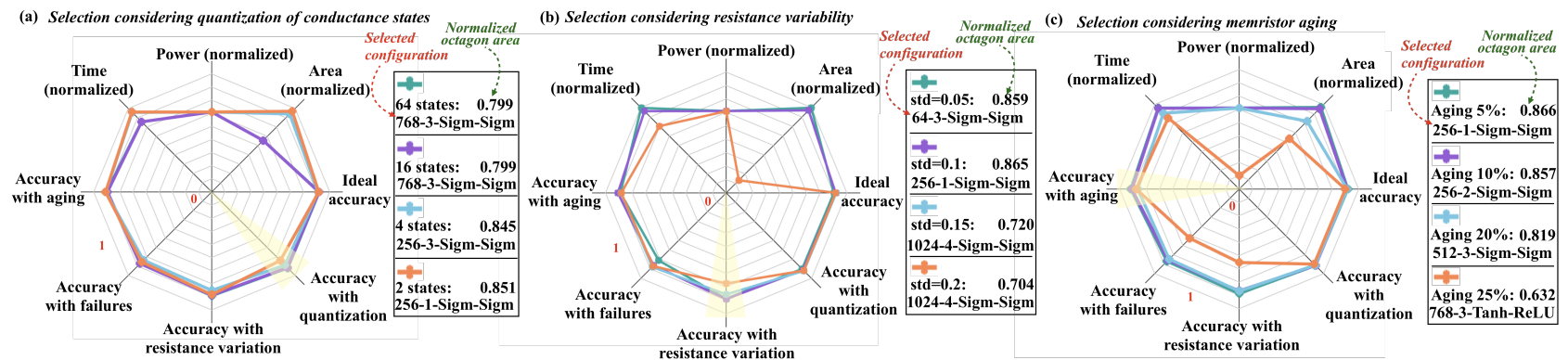

Fig. 3: The selected architectures considering only (a) limited number of stable resistive states (quantization), (b) memristor variation, and (c) memristor aging. In the experiments, only one parameter is varied, while the others are set to $L=16$, $\sigma=0.1, f=2 \%, a=10 \%$ when determining the performance scores.

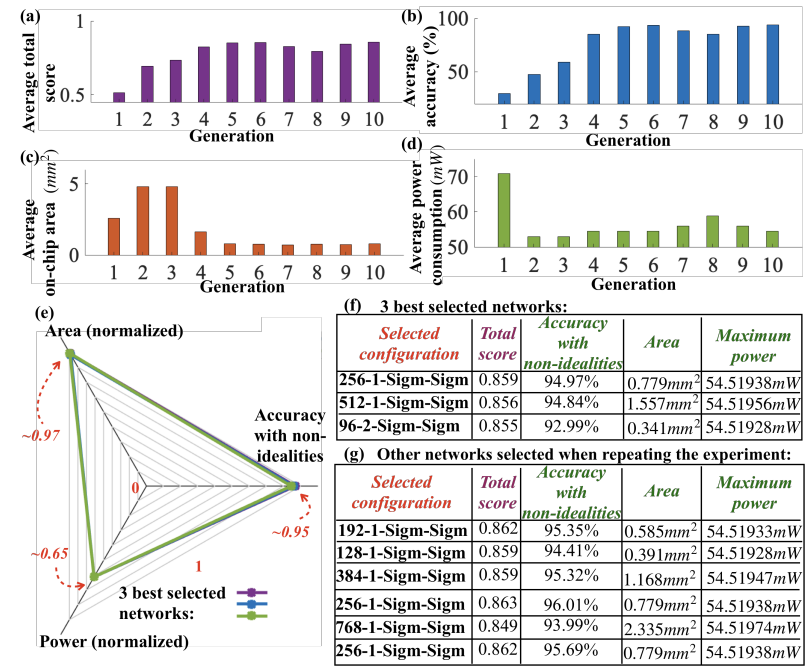

Fig. 4: Average scores per generation of the selected architectures: (a) overall $S$, (b) performance accuracy, (c) on-chip area, (d) power consumption. (e) Overall metrics of 3 best selected architectures after 5 generations. (f) Configurations and parameters of 3 best selected networks. (g) Selected configurations when repeating the experiment for random initialization.

the aging of the devices (Fig. 3 (c)) also tends to select larger architecture when $a$ increases, therefore, normalized octagon area is smaller for higher aging percentage. The selected architectures have the accuracy of $\approx 89 \%$ for $a=5$ $10 \%$ (Fig. 3 (c), cian and purple lines), and $\approx 84 \%$ for $a=20-25 \%$ (Fig. 3 (c), light blue and orange lines). Overall, the experiment showed that it is impossible to completely avoid the reduction of the performance accuracy with nonidealities using considered hyperparameters; while the impact of resistance variation and aging on the performance can be reduced by increasing number of neurons and layers in the architecture. The proposed approach can be applied to any architecture to identify the optimal hyperparameters.

The overall performance of the genetic algorithm is illustrated in Fig. 4. The experiment is performed for MNIST database for $n=[32,48,64,96,128, \ldots 1536,2048]$ and $l=$
$[1,2, \ldots 5]$ and the accuracy is obtained by considering all non-idealities together with $i=25, G=10, p=20$ and $S=\frac{1}{3} P_{s}+\frac{1}{3} A_{s}+\frac{1}{3} A c_{s}$. In this experiment, we consider the maximum number of training epochs before the convergence of accuracy score regardless of the training time. Fig. 4 (ad) illustrates how fast the genetic algorithm converges, where the optimal configuration can be obtained after 5 generations. Fig. 4 (e) shows the overall performance evaluation of 3 best selected networks after 5 generations, where the power score is $\approx 0.65$ and the power is compromised to increase the scores of on-chip area $(\approx 0.97)$ and accuracy $(\approx 0.95)$. Fig. 4 (f) illustrates the configuration of the selected networks and corresponding performance metrics. Several different configurations of the best selected networks can be used to choose the optimum architecture considering the other constraints. For example, out of these 3 networks, the optimum one can be selected based on the crossbar size (number of neurons) preferred by the designer. Fig. 4 (g) illustrates the results of the experiment repeated several times for different sets of random population during initialization stage (when $G=1$ ), showing that the similar optimal architecture is selected regardless of the initialization in the genetic algorithm.

\section{CONCLUSION}

In this paper, we presented the feasibility and impact of taking into account the hardware performance metrics as objective function of genetic search for selecting optimised neural network configuration suitable for a given problem. The memristor crossbar circuits based neural networks are considered as an example tested using MNIST and FashionMNIST dataset. The specific issues such as non-idealities of memristive circuits are taken into account demonstrating the improvements in robustness of selected designs with the proposed multi-objective optimisation approach. The results from Figs. 2-4, indicates that considering hardware non-idealities in optimisation provides better robustness and gives realistic estimate on on-chip performance in area and power, without significantly compromising on the classification accuracy. The algorithmic notion presented in this work can be extended for incorporating any hardware block libraries of neural networks implemented in digital or analog CMOS domain. 


\section{REFERENCES}

[1] J. Bergstra and Y. Bengio, "Random search for hyper-parameter optimization," Journal of Machine Learning Research, vol. 13, no. Feb, pp. 281-305, 2012.

[2] H. Jin, Q. Song, and X. Hu, "Auto-keras: Efficient neural architecture search with network morphism," arXiv preprint arXiv:1806.10282, 2018.

[3] Z. Lu, I. Whalen, V. Boddeti, Y. Dhebar, K. Deb, E. Goodman, and W. Banzhaf, "Nsga-net: neural architecture search using multi-objective genetic algorithm," in Proceedings of the Genetic and Evolutionary Computation Conference. ACM, 2019, pp. 419-427.

[4] H. Mendoza, A. Klein, M. Feurer, J. T. Springenberg, and F. Hutter, "Towards automatically-tuned neural networks," in Workshop on Automatic Machine Learning, 2016, pp. 58-65.

[5] D. Whitley, "A genetic algorithm tutorial," Statistics and computing, vol. 4, no. 2, pp. 65-85, 1994.

[6] K. Deb, A. Pratap, S. Agarwal, and T. Meyarivan, "A fast and elitist multiobjective genetic algorithm: Nsga-ii," IEEE transactions on evolutionary computation, vol. 6, no. 2, pp. 182-197, 2002.

[7] L. Davis, "Handbook of genetic algorithms," 1991.

[8] H. Kitano, "Designing neural networks using genetic algorithms with graph generation system," Complex systems, vol. 4, no. 4, pp. 461-476, 1990.

[9] D. Whitley et al., "Genetic algorithms and neural networks," Genetic algorithms in engineering and computer science, vol. 3, pp. 203-216, 1995.

[10] Y. Li, Z. Wang, R. Midya, Q. Xia, and J. J. Yang, "Review of memristor devices in neuromorphic computing: materials sciences and device challenges," Journal of Physics D: Applied Physics, vol. 51, no. 50, p. 503002, 2018.

[11] W. Ma, F. Caí, C. Du, Y. Jeong, M. Zidan, and W. D. Lu, "Device nonideality effects on image reconstruction using memristor arrays," in 2016 IEEE International Electron Devices Meeting (IEDM). IEEE, 2016, pp. 16-7.

[12] R. Naous, M. Al-Shedivat, and K. N. Salama, "Stochasticity modeling in memristors," IEEE Transactions on Nanotechnology, vol. 15, no. 1, pp. 15-28, 2015.

[13] O. Krestinskaya, A. Irmanova, and A. P. James, "Memristive nonidealities: Is there any practical implications for designing neural network chips?" in 2019 IEEE International Symposium on Circuits and Systems (ISCAS). IEEE, 2019, pp. 1-5.

[14] M. E. Fouda, S. Lee, J. Lee, A. Eltawil, and F. Kurdahi, "Mask technique for fast and efficient training of binary resistive crossbar arrays," IEEE Transactions on Nanotechnology, vol. 18, pp. 704-716, 2019.

[15] J. Xu, Y. Huan, K. Yang, Y. Zhan, Z. Zou, and L.-R. Zheng, "Optimized near-zero quantization method for flexible memristor based neural network," IEEE Access, vol. 6, pp. 29320-29331, 2018.

[16] J. K. Eshraghian, S. Kang, S. Baek, G. Orchard, H. H. Iu, and W. Lei, "Analog weights in reram dnn accelerators," in 2019 IEEE International Conference on Artificial Intelligence Circuits and Systems (AICAS), March 2019, pp. 267-271.

[17] Y. Zhang, G. He, K.-T. Tang, and G. Wang, "On-chip learning of multilayer perceptron based on memristors with limited multilevel states," in 2019 IEEE International Conference on Artificial Intelligence Circuits and Systems (AICAS). IEEE, 2019, pp. 11-12.
[18] B. Liu, H. Li, Y. Chen, X. Li, Q. Wu, and T. Huang, "Vortex: variationaware training for memristor x-bar," in Proceedings of the 52nd Annual Design Automation Conference. ACM, 2015, p. 15.

[19] S. Pal, S. Bose, W.-H. Ki, and A. Islam, "Design of power-and variability-aware nonvolatile rram cell using memristor as a memory element," IEEE Journal of the Electron Devices Society, vol. 7, pp. 701-709, 2019

[20] M. A. Villena, F. Hui, X. Liang, Y. Shi, B. Yuan, X. Jing, K. Zhu, S. Chen, and M. Lanza, "Variability of metal/h-bn/metal memristors grown via chemical vapor deposition on different materials," Microelectronics Reliability, vol. 102, p. 113410, 2019.

[21] S. Smaili and Y. Massoud, "Analytic modeling of memristor variability for robust memristor systems designs," in 2014 IEEE International Symposium on Circuits and Systems (ISCAS). IEEE, 2014, pp. 794-797.

[22] S. Kumar, Z. Wang, X. Huang, N. Kumari, N. Davila, J. P. Strachan, D. Vine, A. D. Kilcoyne, Y. Nishi, and R. S. Williams, "Oxygen migration during resistance switching and failure of hafnium oxide memristors," Applied Physics Letters, vol. 110, no. 10, p. 103503, 2017.

[23] S. Kannan, N. Karimi, R. Karri, and O. Sinanoglu, "Detection, diagnosis, and repair of faults in memristor-based memories," in 2014 IEEE 32nd VLSI Test Symposium (VTS), April 2014, pp. 1-6.

[24] S. Zhang, G. L. Zhang, B. Li, H. H. Li, and U. Schlichtmann, "Aging-aware lifetime enhancement for memristor-based neuromorphic computing," in 2019 Design, Automation \& Test in Europe Conference \& Exhibition (DATE). IEEE, 2019, pp. 1751-1756.

[25] S. N. Mozaffari, K. P. Gnawali, and S. Tragoudas, "An aging resilient neural network architecture," in 2018 IEEE/ACM International Symposium on Nanoscale Architectures (NANOARCH). IEEE, 2018, pp. 1-6.

[26] O. Krestinskaya, K. N. Salama, and A. P. James, "Learning in memristive neural network architectures using analog backpropagation circuits," IEEE Transactions on Circuits and Systems I: Regular Papers, vol. 66, no. 2, pp. 719-732, 2018

[27] K. M. Kim, J. J. Yang, J. P. Strachan, E. M. Grafals, N. Ge, N. D. Melendez, Z. Li, and R. S. Williams, "Voltage divider effect for the improvement of variability and endurance of tao x memristor," Scientific reports, vol. 6, p. 20085, 2016.

[28] L. Deng, "The mnist database of handwritten digit images for machine learning research [best of the web]," IEEE Signal Processing Magazine, vol. 29, no. 6, pp. 141-142, 2012.

[29] H. Xiao, K. Rasul, and R. Vollgraf, "Fashion-mnist: a novel image dataset for benchmarking machine learning algorithms," arXiv preprint arXiv:1708.07747, 2017

[30] D. P. Kingma and J. Ba, "Adam: A method for stochastic optimization," arXiv preprint arXiv:1412.6980, 2014.

[31] T. Chang, "Tungsten oxide memristive devices for neuromorphic applications." 2012.

[32] "The generalized metastable switch memristor model," , Knowm.org, 2018. [Online], available: https://knowm.org/the-generalized-metastableswitch-memristor-model/. [Accessed: 05- Jul- 2019].

[33] F. Cai, J. M. Correll, S. H. Lee, Y. Lim, V. Bothra, Z. Zhang, M. P. Flynn, and W. D. Lu, "A fully integrated reprogrammable memristor-cmos system for efficient multiply-accumulate operations," Nature Electronics, vol. 2, no. 7, pp. 290-299, 2019.

[34] T. Chang, P. Sheridan, and W. Lu, "Modeling and implementation of oxide memristors for neuromorphic applications," in 2012 13th International Workshop on Cellular Nanoscale Networks and their Applications. IEEE, 2012, pp. 1-3. 